\title{
COMPORTAMENTO DE ÓLEOS VEGETAIS EM FRITURAS DESCONTÍNUAS DE PRODUTOS PRÉ-FRITOS CONGELADOS ${ }^{1}$
}

\author{
Patrícia Vieira DEL RÉ ${ }^{2, *}$, Neuza JORGE ${ }^{3}$
}

\begin{abstract}
RESUMO
Este estudo teve como objetivo avaliar algumas alterações de óleos vegetais (girassol, soja e milho), utilizado em sucessivas frituras de produtos pré-fritos congelados (batata palito e produto cárneo empanado (snacks). As frituras dos produtos foram conduzidas em fritadeira doméstica e com as seguintes condições controladas: temperatura de $180^{\circ} \mathrm{C}$, relação superfície/volume de $0,3 \mathrm{~cm}^{-1} \mathrm{e}$ tempo total de aquecimento de $12 \mathrm{~h}$. Nas amostras dos óleos procederam-se as determinações analíticas: compostos polares totais, dienos conjugados, índice de ácido tiobarbitúrico (TBA) e medida da estabilidade oxidativa. Os resultados, em duplicata, obtidos das determinações analíticas foram submetidos às análises de variância, empregando um esquema fatorial, no delineamento inteiramente casual, de modo a determinar a influência dos fatores (produtos, óleos e tempos de fritura) sobre as alterações nos óleos. Os óleos vegetais utilizados nas frituras dos snacks apresentaram menores alterações do que os óleos utilizados para fritura das batatas. Os resultados mostraram que os óleos estudados, apesar das diferenças na composição em ácidos graxos, não apresentaram, em nenhuma análise, valores acima dos limites recomendados em alguns países para o descarte de óleos, independentemente do tipo de produto frito e tempo de aquecimento.
\end{abstract}

Palavras-chave: óleos vegetais, frituras descontínuas, produtos pré-fritos congelados, compostos polares totais.

\section{SUMMARY}

VEGETAL OILS BEHAVIOR IN DISCONTINUED FRYING OF FROZEN PRE-FRIED PRODUCTS. This study was aimed at knowing about the vegetal oils behavior (sunflower, soybean and corn), using consecutive frying of frozen pre-fried products (chip potato and coated meat product - "snacks"). The frying of the products were carried out in a household frying pan under the following controlled conditions: temperature at around $180^{\circ} \mathrm{C}, 0.3 \mathrm{~cm}^{-1}$ surface/volume ratio and $12 \mathrm{~h}$ total heating time. The oil samples were submitted to the analytical determinations: total polar compounds, conjugated dienes, TBA index and oxidative stability. The results obtained from the analytical determinations, in duplicate, were submitted to variance analysis using a factorial scheme on the randomized blocks design, enabling to determine the factors (products, oils and frying time) influence on the oil alteration. The vegetal oils showed lower alteration on the snacks fryings than on the potatoes. The results made evident that the oils studied, despite the difference in the fatty acids composition, did not present at any of the analyses, values over the recommended limits by some countries for oil discard, independently of the fried product type and heating time.

Keywords: vegetal oils, discontinued frying, frozen pre-fried products, total polar compounds.

\section{1 - INTRODUÇÃo}

A alimentação pode refletir-se no equilíbrio psicossomático, pois é parte integrante da saúde física e mental dos indivíduos [21].

Os danos para a saúde que podem decorrer do consumo insuficiente de alimentos - desnutrição, ou do consumo excessivo - obesidade, são há muito tempo conhecidos pelos seres humanos. Apenas mais recentemente, entretanto, acumulam-se evidências de que características qualitativas da dieta são igualmente importantes na definição do estado de saúde, em particular, no que se refere a doenças crônicas da idade adulta [20].

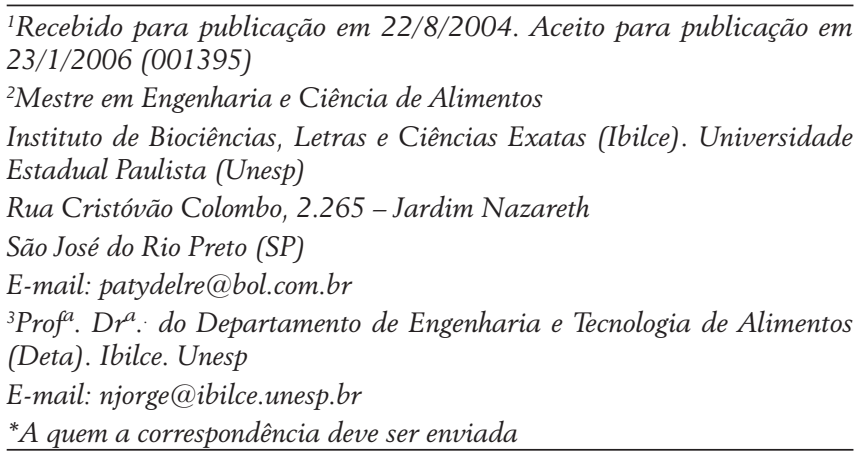

A relevância da ingestão dos óleos vegetais na dieta humana, primordialmente como recursos alimentares provedores de energia, é indiscutível. Entretanto, o risco do desenvolvimento de doenças crônicas decorrentes de seu consumo inadequado, remete a um controle dos aspectos qualitativo e quantitativo dos óleos utilizados nos processos de fritura.

A fritura tem contribuído para o aumento do consumo de óleos e gorduras vegetais, visto que é um processo culinário de grande aceitação em todas as idades e classes sociais [17]. Outro fator é a preferência, no momento da compra, por alimentos que proporcionem facilidade de manipulação e preparo. Estes aspectos fizeram com que as indústrias de alimentos passassem a dispor de produtos específicos para o processo de fritura, como os alimentos pré-fritos congelados, que são largamente adotados pelo mercado consumidor, pois a rapidez é um aspecto fundamental nas sociedades industrializadas atuais.

Durante o processo de fritura por imersão, os óleos são continuamente expostos a vários fatores que levam a uma grande diversidade de reações químicas. Eles podem hidrolisar, formando ácidos graxos livres, monoacilglicerol e diacilglicerol, e/ou oxidar, formando peróxidos, hidroperóxidos, dienos conjugados, epóxidos, hidróxidos 
e cetonas. Podem, ainda, compor-se em pequenos fragmentos ou permanecer na molécula do triacilglicerol, e se associarem, conduzindo a triacilgliceróis diméricos e poliméricos [25, 28].

O nível de alteração depende, sobretudo, das características do alimento, da absorção de ar e da temperatura utilizada e, como conseqüência, a degradação será tanto maior quanto mais prolongado for o período de utilização do óleo ou gordura e quanto maior sua insaturação [8].

Vários critérios são propostos para estabelecer o ponto de descarte de um óleo utilizado em processo de fritura. Alimentos variados são fritos em diferentes tipos de óleos, em diversos tipos de equipamentos e condições de operação. A combinação de todas estas variáveis é que determina a taxa em que as reações de degradação ocorrem. Portanto, um método específico pode ser bom para avaliar em determinado sistema e não ser aplicável a outros [11, 27]. Portanto, é necessário dispor de métodos de controle para avaliar a alteração produzida, assim como, buscar critérios objetivos para definir o ponto em que os óleos devem ser descartados.

Com o decorrer das alterações, as qualidades funcionais, sensoriais e nutricionais dos óleos se modificam, podendo chegar a níveis em que não se consegue mais obter alimentos de qualidade. Estudos têm demonstrado que o consumo excessivo de alimentos fritos representa risco à saúde, possivelmente pela toxicidade dos produtos formados durante o processo de fritura [16].

Entender as mudanças e as alterações que os óleos vegetais sofrem durante o aquecimento, pode ajudar a otimizar os processos de fritura e, conseqüentemente, garantir produtos de melhor qualidade nutricional. Desta forma, os principais objetivos deste estudo foram avaliar algumas alterações de óleos vegetais em frituras descontínuas, utilizando dois produtos distintos: batata palito pré-frita congelada e produto cárneo empanado pré-frito congelado (snacks), e verificar a qualidade e a estabilidade do meio de fritura, a fim de indicar os óleos mais adequados para serem utilizados em processos de fritura e estabelecer o tempo mais adequado para o descarte de cada tipo de óleo utilizado nos processos de fritura.

\section{2 - MATERIAL E MÉTODOS}

\section{1 - Amostras}

Para os testes de frituras descontínuas foram utilizados os seguintes óleos vegetais: óleo de girassol refinado (OGR); óleo de soja refinado (OSR) - que apresentava em sua composição os antioxidantes Terc Butil Hidroquinona (TBHQ) e ácido cítrico - e óleo de milho refinado (OMR), produzidos pela Cargill Agrícola S.A. e provenientes do comércio local.

Os produtos pré-fritos congelados utilizados para as frituras foram batatas palito pré-fritas congeladas e produto cárneo empanado pré-frito congelado - snacks, distribuídos pela Perdigão Agroindustrial S.A., e cedidos para o desenvolvimento do trabalho.

\section{2 - Processo de fritura}

Os ensaios de fritura descontínua foram conduzidos em uma fritadeira doméstica comercial, com capacidade de $3 \mathrm{~L}$. Durante o processo de fritura foram controladas as seguintes condições: temperatura de $180 \pm 2^{\circ} \mathrm{C}$, relação superfície/volume $(\mathrm{s} / \mathrm{v})$ de $0,3 \mathrm{~cm}^{-1}$ e tempo total de experimento de $12 \mathrm{~h}$.

Inicialmente, foram colocados $3 \mathrm{~L}$ de óleo na fritadeira, que foi aquecido por $10 \mathrm{~min}$. Cada lote dos produtos foi frito por $5 \mathrm{~min}$, empregando um intervalo de 30 min entre cada fritura, sendo que 5 min foram utilizados para o reaquecimento do óleo antes de iniciar a fritura seguinte. Foram realizadas as frituras de 25 lotes, cada um com aproximadamente 500 g de produto. Foi empregada reposição de óleo novo nos intervalos das frituras para manter a relação $\mathrm{s} / \mathrm{v}$ constante, correspondendo a uma média de $820 \mathrm{~mL}$, em cada processo de fritura. O período experimental para cada óleo foi de aproximadamente $12 \mathrm{~h}$, divididos em dois dias consecutivos de frituras descontínuas, sendo $6 \mathrm{~h}$ em cada dia. $\mathrm{Na}$ fritura dos snacks foi empregada a filtragem do óleo após as $6 \mathrm{~h}$ iniciais, visto que os snacks são produtos empanados que liberam resíduos.

As amostras de óleo e produtos foram coletadas nos tempos 0,$5 ; 2,5 ; 5,0 ; 7,0 ; 9,5$ e $12 \mathrm{~h}$ de fritura, correspondendo a $\mathrm{F}_{1}, \mathrm{~F}_{5}, \mathrm{~F}_{10}, \mathrm{~F}_{15}, \mathrm{~F}_{20}, \mathrm{~F}_{25}$, respectivamente. O número de fritura foi selecionado, levando-se em consideração os valores das variáveis tipos de produto e óleo, com o objetivo de se obter alterações similares às que se encontram ocasionalmente nos óleos de fritura.

As amostras de óleos $(80 \mathrm{~mL})$ foram coletadas em recipientes de vidro âmbar e os produtos (100 g) foram embaladas em folha de alumínio e, em seguida, armazenadas em freezer à temperatura de aproximadamente $-20^{\circ} \mathrm{C}$, para evitar posteriores alterações oxidativas. As amostras foram descongeladas apenas no momento das análises, as quais foram realizadas em duplicatas.

\section{3 - Determinações analíticas}

- Compostos polares totais - método cromatográfico proposto por DOBARGANES et al. [9], expressos em porcentagem.

- Dienos conjugados - método AOCS Ti 1a-64 [2], que determina dienos conjugados, expressos como porcentagem de ácidos dienóicos conjugados. O espectrofotômetro usado foi da marca Shimadzu, modelo UV mini 1.240.

- Índice de ácido tiobarbitúrico (TBA) - método do Instituto Adolfo Lutz [13]. O espectrofotômetro usado foi da marca Shimadzu, modelo UV mini 1.240, expresso em $\mu$ moles/g.

- Medida da estabilidade oxidativa (Rancimat) - método AOCS Cd 12b-92 [2]. Foi utilizado Rancimat, marca 
Metrohm, modelo 743 , com temperatura de $100^{\circ} \mathrm{C}$ e fluxo de ar de $20 \mathrm{~L} / \mathrm{h}$, sendo o período de indução expresso em horas.

\section{4 - Análise estatística}

Para as determinações analíticas nos óleos foram estabelecidos os seguintes fatores: produtos (batata pré-frita congelada e snacks), óleos (OGR, OSR e OMR) e tempos de fritura $(0,5 ; 2,5 ; 5,0 ; 7,0 ; 9,5$ e 12 h). O experimento foi realizado em um esquema fatorial 2x3x6 com dois produtos, três tipos de óleos e seis tempos de fritura, no delineamento inteiramente casualizado.

Os resultados obtidos das determinações analíticas, em duplicata, foram submetidos à análise de variância para determinar a influência dos fatores sobre a alteração dos óleos submetidos ao processo de fritura, sendo que as diferenças entre as médias foram testadas a $1 \mathrm{e}$ $5 \%$ de probabilidade, pelo teste de Tukey, por meio do programa Sistema para Análises Estatísticas (Estat), versão 2.0, 1999, Brasil.

\section{3 - RESULTADOS E DISCUSSÃo}

A avaliação da alteração e a identificação dos compostos que são formados durante a fritura de alimentos é de grande importância e interesse, não só para pesquisadores, como também para consumidores, indústrias de alimentos e administrações ligadas à saúde pública [24].

Para avaliar os efeitos dos fatores (produtos, óleos e tempos de fritura) sobre as determinações analíticas foram realizados os cálculos das análises de variância (Tabela 1).

Como observado, na Tabela 1, todos os efeitos principais e as interações foram significativos $(p<0,01)$. Então, foi necessário proceder ao desdobramento da interação produtos $\mathrm{x}$ óleos $\mathrm{x}$ tempos de fritura.

\section{1 - Compostos polares}

Entende-se por compostos polares totais todos aqueles compostos que têm uma polaridade maior que os triacil- gliceróis e que correspondem aos não voláteis, resultantes da alteração oxidativa, térmica e hidrolítica [24].

A determinação da quantidade total dos produtos de alteração, originados como conseqüência do processo de fritura, constitui a base das limitações de uso dos óleos existentes em alguns países, estabelecida em torno de 24$27 \%$ de compostos polares [10].

Considerando o limite de descarte acima estabelecido, os óleos estudados apresentaram teores de compostos polares bem abaixo deste limite, sendo maiores os valores médios nas frituras das batatas (12,48\%) quando comparados com os obtidos nas frituras dos snacks $(6,56 \%)$, independentemente do tipo de óleo e tempo de fritura.

Segundo DOBARGANES et al. [7], é importante considerar que alimentos empanados podem ceder partículas da cobertura, as quais contribuem para perdas estéticas do produto, desenvolvendo odores desagradáveis e aumento da degradação do óleo.

Desta forma, em princípio, esperava-se que os snacks, por serem empanados e liberarem partículas durante o processo de fritura, que possivelmente estariam mais sujeitas à carbonização, proporcionassem maior alteração nos óleos. Porém, ao contrário, os óleos da fritura dos snacks apresentaram metade do valor de compostos polares formados quando comparados com os óleos de fritura das batatas. Estes baixos valores podem ser atribuídos ao fato de os produtos serem pré-fritos em gorduras hidrogenadas. Como conseqüência desta fritura prévia, o óleo absorvido se encontra, preferencialmente, na camada externa do alimento, o que facilita as trocas lipídicas e, conseqüentemente, a presença dessas gorduras diminui a alteração do óleo.

Outro aspecto importante é a composição natural em ácidos graxos saturados do produto cárneo (snacks), os quais, durante o processo de fritura, passam a fazer parte do óleo, tornando-o ainda mais resistente às reações termoxidativas e, conseqüentemente, apresentando valores de compostos polares menores em relação aos óleos de fritura das batatas. Observa-se, portanto, que a alteração dos óleos está intimamente relacionada com

TABELA 1 - Análise de variância para as determinações: Compostos Polares Totais (CPT), Dienos Conjugados (DC), Índice de Ácido Tiobarbitúrico (TBA) e Medida da Estabilidade Oxidativa (MEO)

\begin{tabular}{|c|c|c|c|c|c|}
\hline \multirow{2}{*}{ Causas de variação } & \multirow{2}{*}{ G.L. } & \multicolumn{4}{|c|}{ Quadrados médios } \\
\hline & & CPT & DC & TBA $^{1}$ & MEO \\
\hline Produtos & 1 & $632,3753^{* *}$ & $3,8503^{\star *}$ & $1.323,5512^{\star *}$ & $1.076,0934^{* *}$ \\
\hline Óleos & 2 & $3,9960^{* *}$ & $0,2226^{\star *}$ & $66,4310^{\star *}$ & $271,0359^{* *}$ \\
\hline Tempos & 5 & $134,8664^{\star *}$ & $0,7626^{* *}$ & $58,0815^{\star *}$ & $4,0410^{\star *}$ \\
\hline Produtos x óleos & 2 & $5,2495^{\star *}$ & $0,0461^{\star *}$ & $15,9204^{* *}$ & $117,5324^{\star *}$ \\
\hline Produtos $\mathrm{x}$ tempos & 5 & $54,0314^{\star *}$ & $0,2290^{* *}$ & $52,8886^{\star *}$ & $26,0644^{\star *}$ \\
\hline Óleos x tempos & 10 & $0,3631^{* *}$ & $0,0145^{\star *}$ & $30,4963^{* *}$ & $4,2435^{\star *}$ \\
\hline Produtos $\mathrm{x}$ óleos $\mathrm{x}$ tempos & 10 & $0,3464^{* *}$ & $0,0066^{\star *}$ & $15,5857^{* *}$ & $0,9258^{* *}$ \\
\hline Resíduo & 36 & 0,0925 & 0,0001 & 1,2051 & 0,0701 \\
\hline Desvio padrão & & 0,3042 & 0,0094 & 1,0978 & 0,2648 \\
\hline Coeficiente de variação (\%) & & 3,20 & 1,34 & 14,08 & 2,32 \\
\hline
\end{tabular}


seu grau de insaturação, ao afetar fundamentalmente os ácidos graxos insaturados.

Muitos estudos relatam, detalhadamente, a absorção de óleo, o intercâmbio de material de natureza lipídica e a possibilidade de maior adsorção de compostos polares na superfície do produto, durante a fritura de alimentos pré-fritos congelados [22, 24, 26]. Independente da matéria graxa empregada ou do alimento pré-frito submetido à fritura, mais de 90\% do conteúdo lipídico desse alimento, após o preparo, são provenientes do óleo de fritura. Já o alimento pré-frito transfere ao meio de fritura cerca de 85\% do seu conteúdo lipídico inicial [7].

Alguns autores também atribuem os baixos valores de compostos polares à constante reposição de óleo novo empregado nestes processos [4, 18]. Neste trabalho, a reposição com óleo novo foi necessária para repor o óleo absorvido pelo alimento e, assim, manter a relação superfície/volume, contribuindo para os baixos níveis de compostos polares totais.

WARNER et al. [29], analisando o efeito da composição dos ácidos graxos dos óleos de algodão e girassol com alto teor de ácido oléico em frituras de batatas chips e palito, observaram que a deterioração dos óleos usados para a fritura de batatas chips foi bem menor do que a dos óleos usados na fritura de batatas tipo palito, o que pode estar relacionado com a grande reposição de óleo na fritura de batata chips.

A Tabela 2 apresenta as médias de compostos polares, resultado do desdobramento da interação produtos x óleos $\mathrm{x}$ tempos de fritura.

Observa-se na Tabela 2, que os valores de compostos polares totais aumentaram ao longo do tempo de fritura, independentemente dos tipos de produto e óleo utilizados, demonstrando que o aquecimento, mesmo entre períodos curtos foi prejudicial para o óleo. Para os óleos em cada produto, observa-se que os valores tanto para as batatas como para os snacks não diferiram entre si no tempo de fritura $\mathrm{F}_{1}$. Verifica-se, para as batatas, que a partir do tempo fritura $\mathrm{F}_{10}$ o óleo de girassol apresentou maiores valores de compostos polares quando comparados com os óleos de soja e milho. Apesar de estes óleos apresentarem diferenças na composição em ácidos graxos, obtiveram comportamento similar, diferindo-se apenas nos tempos

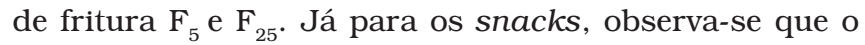
comportamento dos compostos polares foi diferente do encontrado nas batatas, pois a partir do tempo de fritura $\mathrm{F}_{5}$, as porcentagens de compostos polares foram menores para o óleo de milho e, similares entre os óleos de girassol e soja. Em relação aos produtos em cada óleo, observa-se que durante o processo de fritura, as médias de compostos polares para as batatas apresentaram valores maiores em comparação com as médias para os snacks, com exceção do tempo de fritura $\mathrm{F}_{1}$.

Resultados encontrados por DAMY [5] ao estudar o comportamento do óleo de soja em fritura de batatas chips à temperatura de $180^{\circ} \mathrm{C}$, expressam que os valores de compostos polares aumentaram ao longo do tempo de fritura, atingindo $15 \%$ após $7,5 \mathrm{~h}$ de aquecimento.

MASSON et al. [18], estudando o comportamento dos óleos poliinsaturados (soja, girassol, canola) obtidos de estabelecimentos comerciais, em frituras de batatas palito, também obtiveram resultados abaixo da recomendação para os compostos polares totais, variando de 5,3 a $21,3 \%$.

\section{2 - Dienos conjugados}

Quando os ácidos graxos dos óleos poliinsaturados são oxidados, principalmente ácidos linoléico e linolênico, formam-se dienos conjugados que podem ser medidos por absorção ultravioleta a 232-234 nm [12, 19, 30].

O acompanhamento dos espectros de absorção na faixa do ultravioleta das amostras de óleo fornece uma boa indicação das alterações que ocorrem durante o processo oxidativo, visto que o índice de peróxidos não reflete o aumento da degradação do óleo com o tempo de fritura. Por serem instáveis, os peróxidos são rapidamente formados e quebrados em compostos menores, enquanto os dienos conjugados que se formam concomitantemente, permanecem no óleo de fritura [3].

A Tabela 3 apresenta as médias de dienos conjugados, resultado do desdobramento da interação produtos x óleos $\mathrm{x}$ tempos de fritura.

TABELA 2 - Médias de compostos polares (\%), para cada combinação de produtos, óleos e tempos de fritura

\begin{tabular}{|c|c|c|c|c|c|c|}
\hline \multirow{2}{*}{ Tempos de fritura (h) } & \multicolumn{3}{|c|}{ Batatas } & \multicolumn{3}{|c|}{ Snacks } \\
\hline & OGR & OSR & OMR & OGR & OSR & OMR \\
\hline$F_{1}(0,5)$ & $4,78^{\mathrm{fA \alpha} \alpha}$ & $4,81^{\mathrm{fA} \alpha}$ & $4,72^{\mathrm{fA} \alpha}$ & $4,60^{\mathrm{dA} \beta}$ & $5,07^{\mathrm{eA} \beta}$ & $4,87^{\mathrm{eA \beta}}$ \\
\hline$F_{5}(2,5)$ & $8,49^{\mathrm{eA \alpha}}$ & $7,27^{\mathrm{eB} \alpha}$ & $8,31^{\mathrm{eA} \alpha}$ & $5,94^{\mathrm{cAB}}$ & $5,89^{\text {deA } \beta}$ & $5,06^{\mathrm{deB} \beta}$ \\
\hline$F_{10}(5,0)$ & $12,27^{\mathrm{dA} \alpha}$ & $10,89^{\mathrm{dB} \alpha}$ & $11,26^{\mathrm{dB} \alpha}$ & $6,37^{\mathrm{bcA} \beta}$ & $6,68^{\mathrm{cdA} \beta}$ & $5,56^{\mathrm{cdB} \beta}$ \\
\hline $\mathrm{F}_{15}(7,0)$ & $15,90^{\mathrm{cA \alpha}}$ & $13,35^{\mathrm{cB} \alpha}$ & $13,50^{\mathrm{cB} \alpha}$ & $6,84^{\mathrm{bAB} \beta}$ & $7,42^{\mathrm{bcA} \beta}$ & $6,42^{\mathrm{bcB} \beta}$ \\
\hline $\mathrm{F}_{20}(9,5)$ & $18,50^{\mathrm{bA} \alpha}$ & $16,75^{\mathrm{bB} \alpha}$ & $17,14^{\mathrm{bB} \alpha}$ & $7,85^{\mathrm{aA} \beta}$ & $8,25^{\mathrm{abA} \beta}$ & $7,04^{\mathrm{abB} \beta}$ \\
\hline $\mathrm{F}_{25}(12,0)$ & $19,93^{\mathrm{aA \alpha}}$ & $17,86^{\mathrm{aC} \alpha}$ & $18,98^{\mathrm{aB} \alpha}$ & $8,29 \mathrm{aA} \beta$ & $8,37^{\mathrm{aA} \beta}$ & $7,50^{\mathrm{aB} \beta}$ \\
\hline
\end{tabular}

OGR - Óleo de Girassol Refinado. OSR - Óleo de Soja Refinado. OMR - Óleo de Milho Refinado. a,bEm cada coluna, médias dos tempos de fritura seguidas de mesma letra minúscula, não diferem entre si pelo teste de Tukey $(\mathrm{P}>0,05)$. A.BEm cada linha, médias dos óleos em cada produto seguidas de mesma letra maiúscula, não diferem entre si pelo teste de Tukey $(\mathrm{P}>0,05)$. ${ }^{\alpha, \beta} \mathrm{Em}$ cada linha, médias dos produtos em cada óleo seguidas de mesma letra grega, não diferem entre si pelo teste de Tukey $(\mathrm{P}>0,05)$ 
Observa-se na Tabela 3, em relação aos tempos de fritura, que a formação de dienos conjugados foi crescente durante o aumento do tempo de aquecimento, independentemente dos tipos de produto e óleo utilizados. Para os óleos em cada produto, observa-se que, no processo de fritura das batatas, a partir do tempo de fritura $\mathrm{F}_{5}$, o óleo de girassol apresentou maior porcentagem de dienos conjugados, seguidos pelos óleos de soja e milho. Já nas frituras dos snacks, a maior concentração de dienos conjugados variou entre os óleos de girassol e soja, e manteve-se mais baixa no óleo de milho. Em relação aos produtos em cada tipo óleo, verifica-se que a formação de dienos conjugados foi maior durante a fritura das batatas do que nas frituras do snacks, independentemente do tipo de óleo.

Os valores de dienos conjugados obtidos neste trabalho não foram similares aos encontrados por outros autores, LAKE \& SCHOLES [14], os quais demonstraram que produtos como carnes e peixes apresentaram valores de dienos conjugados superiores a produtos de origem vegetal, como as batatas fritas. Este fato se deve, às diferenças existentes no procedimento de fritura no que se refere ao tempo de fritura e tipo de produto.

Todavia, os valores encontrados neste trabalho foram similares aos descritos por MASSON et al. [18], que estudaram o comportamento de óleos poliinsaturados obtidos de estabelecimentos comerciais, em frituras de batatas, os quais apresentaram-se dentro do intervalo 0,5 a $2,6 \%$.

DEL RÉ et al. [6], em estudo do comportamento do óleo de soja refinado em frituras descontínuas de batata palito, constataram que o acréscimo do tempo de aquecimento promoveu um aumento nos valores de dienos conjugados.

\section{3 - Índice de ácido tiobarbitúrico (TBA)}

A Tabela 4 apresenta as médias de índice de TBA, resultado do desdobramento da interação produtos $\mathrm{x}$ óleos $\mathrm{x}$ tempos de fritura.

Segundo os dados da Tabela 4, em relação aos tempos de fritura, para as batatas, verifica-se que os índices de TBA tiveram um comportamento instável oscilando entre um aumento e uma diminuição. Já, para os snacks, constata-se que os valores encontrados nos óleos de girassol e soja diminuíram com o tempo de fritura, e no óleo de milho oscilou entre um aumento e uma diminuição. Para os óleos em cada produto, observa-se que no processo de fritura dos snacks, os valores do índice de TBA não diferem entre si no tempo de fritura $F_{1}$. Em relação aos produtos em cada tipo de óleo, observa-se que o índice de TBA é maior nas frituras das batatas do que os valores obtidos nas frituras do snacks, independentemente do tipo de óleo e tempo de fritura. A exceção foi o tempo de fritura $\mathrm{F}_{1}$, no óleo de girassol da fritura dos snacks, o qual não diferiu, significativamente, da fritura das batatas. O mesmo ocorreu com o óleo de milho no tempo de fritura $\mathrm{F}_{5}$.

O fato de os valores de TBA encontrados nas batatas serem maiores do que os valores dos snacks, pode ser atribuído ao grau de insaturação, visto que os ácidos graxos saturados dos snacks, durante o processo de fritura,

TABELA 3 - Médias de dienos conjugados (\%), para cada combinação de produtos, óleos e tempos de fritura

\begin{tabular}{|c|c|c|c|c|c|c|}
\hline \multirow{2}{*}{ Tempos de fritura $(\mathrm{h})$} & \multicolumn{3}{|c|}{ Batatas } & \multicolumn{3}{|c|}{ Snacks } \\
\hline & OGR & OSR & OMR & OGR & OSR & OMR \\
\hline$F_{1}(0,5)$ & $0,342^{\mathrm{fB} \alpha}$ & $0,397^{\mathrm{fA \alpha} \alpha}$ & $0,287^{\mathrm{fC} \alpha}$ & $0,285^{\mathrm{fB} \beta}$ & $0,399^{\mathrm{e} A \beta}$ & $0,216^{\mathrm{dC} \beta}$ \\
\hline$F_{5}(2,5)$ & $0,658^{\mathrm{eA \alpha}}$ & $0,629^{\mathrm{eB} \alpha}$ & $0,591^{\mathrm{eC} \alpha}$ & $0,419^{\mathrm{eB} \beta}$ & $0,451^{\mathrm{d} A \beta}$ & $0,330^{\mathrm{cc} \beta}$ \\
\hline$F_{10}(5,0)$ & $1,043^{\mathrm{dA} \alpha}$ & $0,916^{\mathrm{dB} \alpha}$ & $0,807^{\mathrm{dC} \alpha}$ & $0,466^{\mathrm{dB} \beta}$ & $0,506^{\mathrm{cA} \beta}$ & $0,411^{\mathrm{bc} \beta}$ \\
\hline $\mathrm{F}_{15}(7,0)$ & $1,341^{\mathrm{cA \alpha}}$ & $1,056^{\mathrm{cB} \alpha}$ & $0,917^{\mathrm{cC} \alpha}$ & $0,561^{\mathrm{cA} \beta}$ & $0,517^{\mathrm{cB} \beta}$ & $0,429^{\mathrm{bc} \beta}$ \\
\hline $\mathrm{F}_{20}(9,5)$ & $1,495^{\mathrm{bA \alpha}}$ & $1,170^{\mathrm{bB} \alpha}$ & $1,040^{\mathrm{bC} \alpha}$ & $0,600^{\mathrm{bA} \beta}$ & $0,601^{\mathrm{bA} \beta}$ & $0,474^{\mathrm{aB} \beta}$ \\
\hline$F_{25}(12,0)$ & $1,552^{\mathrm{aA} \alpha}$ & $1,338^{\mathrm{aB} \alpha}$ & $1,237^{\mathrm{aC} \alpha}$ & $0,726^{\mathrm{aA} \beta}$ & $0,640^{\mathrm{aB} \beta}$ & $0,487^{\mathrm{ac} \beta}$ \\
\hline
\end{tabular}

OGR - Óleo de Girassol Refinado. OSR - Óleo de Soja Refinado. OMR - Óleo de Milho Refinado. a.bEm cada coluna, médias dos tempos de fritura seguidas de mesma letra minúscula, não diferem entre si pelo teste de Tukey $(\mathrm{p}>0,05)$. ${ }^{\mathrm{A} B \mathrm{~B}} \mathrm{Em}$ cada linha, médias dos óleos em cada produto seguidas de mesma letra maiúscula, não diferem entre si pelo teste de Tukey $(p>0,05)$. ${ }^{\alpha . \beta} \mathrm{Em}$ cada linha, médias dos produtos em cada óleo seguidas de mesma letra grega, não diferem entre si pelo teste de Tukey ( $\left.\mathrm{p}>0,05\right)$

TABELA 4 - Médias de índice de TBA ( $\mu$ moles/g), para cada combinação de produtos, óleos e tempos de fritura.

\begin{tabular}{|c|c|c|c|c|c|c|}
\hline \multirow{2}{*}{ Tempos de fritura (h) } & \multicolumn{3}{|c|}{ Batatas } & \multicolumn{3}{|c|}{ Snacks } \\
\hline & OGR & OSR & OMR & OGR & OSR & OMR \\
\hline $\mathrm{F}^{1}(0,5)$ & $0,0047^{\mathrm{cC} \alpha}$ & $0,0173^{\mathrm{bA \alpha}}$ & $0,0114^{\mathrm{abB} \alpha}$ & $0,0039 \mathrm{aA} \beta$ & $0,0055 a A \beta$ & $0,0055 b A \beta$ \\
\hline$F^{5}(2,5)$ & $0,0071^{\mathrm{cB} \alpha}$ & $0,0122^{\mathrm{cA \alpha} \alpha}$ & $0,0126^{\mathrm{aA \alpha}}$ & $0,0012 a b C \beta$ & $0,0063 a \mathrm{aB} \beta$ & $0,0110 \mathrm{aA} \beta$ \\
\hline$F^{10}(5,0)$ & $0,0244^{\mathrm{aA \alpha}}$ & $0,0220^{\mathrm{aA} \alpha}$ & $0,0138^{\mathrm{aB} \alpha}$ & $0,0016 a b B \beta$ & $0,0055 a A \beta$ & $0,0035 \mathrm{bcAB} \beta$ \\
\hline $\mathrm{F}^{15}(7,0)$ & $0,0075^{\mathrm{cB} \alpha}$ & $0,0158^{\mathrm{bA \alpha}}$ & $0,0087^{\mathrm{bB} \alpha}$ & $0,0000 \mathrm{bB} \beta$ & $0,0043 a b A \beta$ & $0,0008 \mathrm{cB} \beta$ \\
\hline $\mathrm{F}^{20}(9,5)$ & $0,0098^{\mathrm{bcA} \alpha}$ & $0,0087^{\mathrm{dA} \alpha}$ & $0,0106^{\mathrm{abA \alpha}}$ & $0,0000 \mathrm{bB} \beta$ & $0,0039 a b A \beta$ & $0,0039 \mathrm{bA} \beta$ \\
\hline $\mathrm{F}^{25}(12,0)$ & $0,0110^{\mathrm{bA} \alpha}$ & $0,0071^{\mathrm{dB} \alpha}$ & $0,0130^{\mathrm{aA} \alpha}$ & $0,0000 \mathrm{bB} \beta$ & $0,0012 b B \beta$ & $0,0051 \mathrm{bA} \beta$ \\
\hline
\end{tabular}

OGR - Óleo de Girassol Refinado. OSR - Óleo de Soja Refinado. OMR - Óleo de Milho Refinado. a,bEm cada coluna, médias dos tempos de fritura seguidas de mesma letra minúscula, não diferem entre si pelo teste de Tukey $(\mathrm{p}>0,05)$. A.B Em cada linha, médias dos óleos em cada produto seguidas de mesma letra maiúscula, não diferem entre si pelo teste de Tukey $(\mathrm{p}>0,05) .{ }^{\alpha / \beta} \mathrm{Em}$ cada linha, médias dos produtos em cada óleo seguidas de mesma letra grega, não diferem entre si pelo teste de Tukey ( $>0,05$ ) 
TABELA 5 - Médias da medida da estabilidade oxidativa (horas), para cada combinação de produtos, óleos e tempos de fritura

\begin{tabular}{|c|c|c|c|c|c|c|}
\hline \multirow{2}{*}{ Tempos de fritura (h) } & \multicolumn{3}{|c|}{ Batatas } & \multicolumn{3}{|c|}{ Snacks } \\
\hline & OGR & OSR & OMR & OGR & OSR & OMR \\
\hline$F_{1}(0,5)$ & $7,43^{\mathrm{ac} \beta}$ & $11,45^{\mathrm{aB} \beta}$ & $13,48^{\mathrm{aA} \beta}$ & $9,94^{\mathrm{eB} \alpha}$ & $9,79^{\text {ев } \alpha \beta}$ & $22,86^{\mathrm{Aa} \alpha}$ \\
\hline $\mathrm{F}_{5}(2,5)$ & $5,64^{\mathrm{bB} \beta}$ & $9,98^{\mathrm{bA \beta}}$ & $10,49^{\mathrm{b} A \beta}$ & $11,39^{\mathrm{dB} \alpha}$ & $11,19^{\mathrm{dB} \alpha}$ & $20,00^{\mathrm{dA} \alpha}$ \\
\hline$F_{10}(5,0)$ & $4,27^{\mathrm{cC} \beta}$ & $9,57 b^{c A B}$ & $8,86^{\mathrm{cB} \beta}$ & $12,21^{\mathrm{cB} \alpha}$ & $12,35^{\mathrm{cB} \alpha}$ & $21,30^{\mathrm{bA \alpha}}$ \\
\hline$F_{15}(7,0)$ & $3,30^{\mathrm{dB} \beta}$ & $8,89^{\mathrm{cA \beta}}$ & $8,87^{\mathrm{CA \beta}}$ & $12,78^{\mathrm{bcB} \alpha}$ & $12,93^{\mathrm{cB} \alpha}$ & $19,60^{\mathrm{dA} \alpha}$ \\
\hline$F_{20}(9,5)$ & $2,83^{\mathrm{de} C \beta}$ & $7,98^{\mathrm{dA} \beta}$ & $6,19^{\mathrm{dB} \beta}$ & $13,49^{\mathrm{abB} \alpha}$ & $13,98^{\mathrm{bB} \alpha}$ & $20,98^{\mathrm{CA \alpha}}$ \\
\hline
\end{tabular}

OGR - Óleo de Girassol Refinado. OSR - Óleo de Soja Refinado. OMR - Óleo de Milho Refinado. a,bEm cada coluna, médias dos tempos de fritura seguidas de mesma letra minúscula, não diferem entre si pelo teste de Tukey $(\mathrm{p}>0,05)$. A.B Em cada linha, médias dos óleos em cada produto seguidas de mesma letra maiúscula, não diferem entre si pelo teste de Tukey $(\mathrm{p}>0,05)$. a,bem cada linha, médias dos produtos em cada óleo seguidas de mesma letra grega, não diferem entre si pelo teste de Tukey ( $>0,05$ )

passam a fazer parte do óleo, contribuindo para diminuir os níveis de TBA, os quais são evidenciados à medida que aumenta o tempo de fritura, chegando a alcançar valor igual a zero a partir da fritura $\mathrm{F}_{15}$ no óleo de girassol.

LAKE \& SCHOLES [14], em experimentos com frituras de batatas pré-fritas congeladas em óleos vegetais, encontraram valores de TBA que variavam entre 0,07 e $0,22 \mu$ moles/g, valores estes superiores aos encontrados neste trabalho. Em outro experimento similar, ZHANG \& ADDIS [31], determinando o índice de TBA de uma mistura de $90 \%$ de sebo e $10 \%$ de óleo de algodão, utilizada para fritura de batatas chips a aproximadamente $170^{\circ} \mathrm{C}$, encontraram valores na faixa de 0,004 a 0,010 $\mu$ moles $/ g$.

\section{4 - Medida da estabilidade oxidativa}

A estabilidade de óleos é definida como o tempo para se atingir nível de rancidez detectável ou surpreendente mudança na taxa de oxidação [1]. O método do Rancimat baseia-se na determinação da condutividade elétrica dos produtos voláteis de degradação. Por esse método, uma curva de condutividade elétrica x tempo é automaticamente registrada com o decorrer da reação e do teste, o período de indução é determinado em horas.

A Tabela 5 apresenta as médias da medida da estabilidade oxidativa, resultado do desdobramento da interação produtos $\mathrm{x}$ óleos $\mathrm{x}$ tempos de fritura.

Como observado na Tabela 5, em relação aos tempos de fritura, observa-se uma redução do período de indução, conforme o aumento do tempo de aquecimento nos processos de fritura das batatas. Já para os snacks, observa-se o oposto - o período de indução aumenta com o tempo de fritura, fator este, também relacionado à quantidade de ácidos graxos saturados liberados durante o processo de fritura. Estes valores reforçam o fato de que a estabilidade oxidativa está igualmente relacionada com o grau de insaturação do óleo ou gordura.

Quanto aos óleos em cada tipo de produto, observa-se que, nas frituras das batatas, o óleo de girassol apresentou o menor período de indução, valor este que pode ser correlacionado com a maior quantidade de compostos polares formados neste óleo durante o processo de fritura. Já o óleo de soja apresentou os menores valores, podendo estar atribuído à presença dos antioxidantes TBHQ e ácido cítrico.
Nas frituras dos snacks, o maior período de indução corresponde ao óleo de milho, o qual apresentou os menores valores de compostos polares. Os demais óleos apresentaram comportamento similar, diferindo apenas na fritura $\mathrm{F}_{25}$.

Para os produtos, verifica-se que os períodos de indução durante as frituras das batatas foram menores que os valores obtidos para as frituras dos snacks, com exceção do óleo de soja no tempo de fritura $F_{1}$, o qual apresentou maior valor na fritura das batatas.

Em geral, observa-se uma relação direta entre a estabilidade oxidativa dos óleos, os compostos polares e o grau de insaturação no comportamento dos óleos durante a fritura.

\section{5 - Correlações}

A Tabela 6 apresenta os coeficientes de correlação entre os testes analíticos realizados nos óleos de fritura, considerando os dois produtos separadamente.

Segundo PÉREZ-CAMINO et al. [23], a separação das amostras em grupos mais homogêneos é útil para explicar as diferenças existentes entre distintos autores, devido a que, deste modo, reduz-se o número de variáveis envolvidas no processo.

De acordo com resultados da Tabela 6 , destaca-se a correlação encontrada entre a porcentagem de compostos polares totais e dienos conjugados, em ambos os produtos. MASSON et al. [18] encontraram correlação entre estes índices de 0,64; já, LOPES [15] encontrou 0,72. Diferenças distantes podem ser explicadas pelas características dos óleos e pelas diferentes condições em que ocorreram as frituras.

TABELA 6 - Coeficientes de correlação entre os testes analíticos realizados nos óleos de fritura

\begin{tabular}{ccccc}
\hline & CPT & DC & TBA & MEO \\
\hline Batatas & 1,00 & & & \\
CPT & $0,92^{*}$ & 1,00 & & \\
DC & $-0,13$ & $-0,03$ & 1,00 & \\
TBA & $-0,69^{*}$ & $-0,72^{*}$ & 0,16 & 1,00 \\
MEO & & & \\
\hline Snacks & 1,00 & & & \\
CPT & $0,91^{*}$ & 1,00 & & 1,00 \\
DC & $-0,49^{*}$ & $-0,57^{*}$ & 1,00 & 0,24 \\
TBA & $-0,04$ & $-0,28$ & & \\
MEO & & & \\
\hline CPT - Compostos Polares Totais. DC - Dienos Conjugados. TBA - Índice de Ácido \\
Tiobarbitúrico. MEO - Medida da Estabilidade Oxidativa. *Teste sionnificativo (p<0,05)
\end{tabular}


Na Tabela 7, os coeficientes de correlação entre as determinações analíticas foram determinados considerando os dados referentes ao tempo de fritura $\mathrm{F}_{25}$. Observa-se que quando estabelecido o tempo de fritura, os coeficientes de correlação lineares entre os métodos analíticos melhoraram.

Observando as correlações entre o tempo de fritura $\mathrm{F}_{25}$, destacam-se os altos valores de correlação inversa entre a medida da estabilidade oxidativa e as análises de compostos polares, demonstrando que à medida que se formam compostos de degradação, diminui a estabilidade oxidativa dos óleos.

TABELA 7 - Coeficientes de correlação entre os testes analíticos realizados nos óleos referentes ao tempo de fritura $\mathrm{F}_{25}$.

\begin{tabular}{|c|c|c|c|c|}
\hline & CPT & DC & TBA & MEO \\
\hline \multicolumn{5}{|l|}{ Batatas } \\
\hline $\mathrm{CPT}$ & 1,00 & & & \\
\hline $\mathrm{DC}$ & 0,62 & 1,00 & & \\
\hline TBA & 0,63 & $-0,14$ & 1,00 & \\
\hline MEO & $-0,96^{*}$ & $-0,75$ & $-0,55$ & 1,00 \\
\hline \multicolumn{5}{|l|}{ Snacks } \\
\hline CPT & 1,00 & & & \\
\hline $\mathrm{DC}$ & 0,80 & 1,00 & & \\
\hline TBA & $-0,77$ & $-0,96^{*}$ & 1,00 & \\
\hline MEO & $-0,86^{*}$ & $-0,97^{*}$ & 0,98 & 1,00 \\
\hline
\end{tabular}

\section{4 - CONCLUSÕES}

Em resumo, os resultados obtidos neste estudo permitem as seguintes conclusões:

- Os óleos vegetais utilizados neste estudo apresentaram menores alterações nas frituras dos snacks do que nas frituras das batatas, fato que pode ser atribuído à composição, em ácidos graxos presentes no produto cárneo;

- Os óleos analisados, girassol, soja e milho, apesar das diferenças na composição em ácidos graxos, não apresentaram, na análise, valores acima dos limites recomendados em alguns países para o descarte dos mesmos, independentemente do tipo de produto frito e tempo de aquecimento;

- As condições estabelecidas no processo de fritura: tempo e temperatura de aquecimento, relação s/v e reposição de óleo novo foram seguras, garantindo produtos de qualidade e indicando que todos os óleos foram adequados à fritura, mesmo os óleos de girassol e milho que não apresentavam antioxidantes sintéticos;

- Quanto às determinações analíticas, observou-se uma relação direta entre a estabilidade oxidativa e os compostos polares, formados ao longo do processo de fritura dos produtos.

\section{5 - REFERÊNCIAS BIBLIOGRÁFICAS}

[1] ANTONIASSI, R. Métodos de avaliação da estabilidade oxidativa de óleos e gorduras. Boletim do Centro de Pesquisa e Processamento de Alimentos. Curitiba, v. 19, n. 2, p. 353-380, 2001.

[2] AOCS. Official methods and recommended practices of the American Oil Chemists' Society. Champaign: AOCS, 1993.

[3] CELlA, R. C. F.; REGITANO-D'ARCE, M.A.B.; SPOTO, M.H.F. Comportamento do óleo de soja refinado utilizado em fritura por imersão com alimentos de origem vegetal. Cienc. Tecnol. Aliment., v. 22, n. 2, p. 111-116, 2002.

[4] CUESTA, C.; SÁNCHEZ-MUNIZ, F.J. Quality control during repeated fryings. Grasas y Aceites, v. 49, n. 3-4, p. 310-318, 1998.

[5] DAMY, P.C. Alterações físico-químicas de óleo de soja e da gordura hidrogenada durante o processo de fritura descontínua de batatas. 2001. $136 \mathrm{f}$. Dissertação (mestrado em Engenharia e Ciência de Alimentos). Instituto de Biociências, Letras e Ciências Exatas (Ibilce). Universidade Estadual Paulista (Unesp), São José do Rio Preto (SP).

[6] DEL RÉ, P.V.; COLTRO, A.L.; MANENTE, J.C.P.P.; MARTI, G.E.; JORGE, N. Influência da relação superfície/volume em frituras de batata palito. Rev. Inst. Adolfo Lutz, v. 62, n. 3, p. 213-219, 2003.

[7] DOBARGANES, M.C.; MÁRgUEZ-RUIZ, G.; VELASCO, $J$. Interactions between fat and food during deep-frying. Eur. J. Lipid Sci. Technol., v. 102, p. 521-528, 2000.

[8] DOBARGANES, M.C.; PÉREZ-CAMINO, M.C.; MÁRQUEZ-RUIZ, G. Determinación de compuestos polares en aceites y grasas de fritura. Grasas y Aceites, v. 40, n. 1, p. 35-38, 1989.

[9] DOBARGANES, M.C.; VELASCO, J., DIEFFENBACHER, A. Determination of polar compounds, polymerized and oxidized triacylglycerols, and diacylglycerols in oils and fats. Pure Appl. Chem., v. 72, n. 8, p. 1563 $1575,2000$.

[10] FIRESTONE, D.; STIER, R.F.; BLUMENTHAL, M.M. Regulation of frying fats and oils. Food Technol., v. 45, n. 2, p. 90-94, 1991.

[11] FRITSCH, C.W. Measurements of frying fat deterioration: a brief review. J. Am. Oil Chem. Soc., v. 58, n. 3, p. 272-274, 1981.

[12] GUTIÉRREZ, R.G.; DOBARGANES, M.C. Analytical procedures for the evaluation of used frying fats. In: VARELA, G.; BENDER, A.E.; MORTON, I.A. (ed.) Frying foods: principles, changes, new approaches. Chichester. Ellis Horwood, p. 141-154. 1988.

[13] INSTITUTO ADOLFO LUTZ. São Paulo. Normas analíticas do Instituto Adolfo Lutz. São Paulo, I.O.E., p. 245-266, 1985.

[14] LAKE, R.J.; SCHOLES, P. Quality and consumption of oxidized lipids from deep-frying fats and oils in New Zealand. J. Am. Oil Chem. Soc., v. 74, n. 9, p. 1.065-1.068, 1997.

[15] LOPES, M.R.V. Estudo comparativo entre métodos analíticos tradicionais e testes rápidos utilizados na avaliação da qualidade dos óleos e gorduras de fritura. 2002. 136 f. Dissertação (mestrado em Enge- 
nharia e Ciência de Alimentos) - Instituto de Biociências, Letras e Ciências Exatas (Ibilce). Universidade Estadual Paulista (Unesp), São José do Rio Preto (SP).

[16] MÁRQUEZ-RUIZ, G.; PÉREZ-CAMINO, M.C.; DOBARGANES, M.C. Evaluación nutricional de grasas termoxidadas y de fritura. Grasas y Aceites, v. 41, p. 432-439, 1990.

[17] MASSON, L.; ROBERT, P.; IZAURIETA, M.; ROMERO, N.; ORTIZ, J. Fat deterioration in deep fat frying french fries potatoes at restaurant and food shop sector. Grasas y Aceites, v. 50, n. 6, p. 460-468, 1999.

[18] MASSON, L.; ROBERT, P.; ROMERO, N.; IZAURIETA, M.; VALENZUELA, S.; ORTIZ, J.; DOBARGANES, M.C. Comportamiento de aceites poliinsaturados en la preparación de patatas fritas para consumo inmediato: Formación de nuevos compuestos y comparación de métodos analíticos. Grasas y Aceites, v. 48, n. 5, p. 273-281, 1997.

[19] MELTON, S.L.; JAFAR, S.; SYKES, D.; TRIGIANO, M.K. Review of stability measurements for frying oils and fried food flavor. J. Am. Oil Chem. Soc., v. 71, p. 1.301-1.308, 1994.

[20] MONTEIRO, C.A.; MONDINI, B.; COSTA, R.B.L. Mudanças na composição e adequação nutricional da dieta familiar nas áreas metropolitanas do Brasil (1988-1996). Rev. de Saúde Pública, v. 34, n. 3, p. 1-13, 2000.

[21] PASTORE, C.A. A saúde do coração na alimentação. Higiene Alimentar, v. 12, n. 55, p. 13, maio/jun., 1998.

[22] PÉREZ-CAMINO, M.C.; MÁRQUEZ-RUIZ, G.; RUÍZMÉNDEZ, M.V.; DOBARGANES, M.C. Lipid changes during frying of frozen prefried foods. J. Food Sci., v. 56, p. 1.644-1.647, 1991.

[23] PÉREZ-CAMINO, M.C.; MÁRQUEZ-RUIZ, G.; SALGADO-RAPOSO, A.; DOBARGANES, M.C. Alteración de grasas usadas en fritura. III. Correlación entre índices analíticos y métodos de evaluación directa de compuestos de degradación. Grasas y Aceites, v. 39, n. 2, p. 72-76, 1988.
[24] POZO-DÍEZ, R.M. Estudio del proceso de fritura de alimentos frescos y congelados prefritos. Comportamiento del aceite de semilla de girasol de alto contenido en ácido oleico. 1995. 338 f. Tese (doutorado em Farmácia). Facultad de Farmacia. Universidad de Alcalá de Henares, Alcalá de Henares.

[25] SANIBAL, E.A.A.; MANCINI-FILHO, J. Alterações físicas, químicas e nutricionais de óleos submetidos ao processo de fritura. Food Ingred. South Am., v. 18, p. 64-71, 2002.

[26] SEBÉDIO J.L.; BONPUNT, A.; GRANDGIRAD, A.; PREVOST, J. Deep fat frying of frozen prefried french fries: Influence of the amount of linoleic acid in the frying medium. J. Agric. Food Chem., v. 38, n. 9, p. 1.862-1.867, 1990.

[27] STEVENSON, S.G.; VAISEY-GENSER, M.; ESKIN, N.A.M. Quality control in the use of deep frying oils. J. Am. Oil Chem. Soc., v. 61, n. 6, p. 1.102$1.108,1984$.

[28] TAKEOKA, G.R.; FULL, G.H.; DAO, L.T. Effect of heating on the characteristics and chemical composition of selected frying oils and fats. J. Am. Oil Chem. Soc., v. 45, p. 3.244-3.249, 1997.

[29] WARNER, K.; ORR, P.; GLYNN, M. Effect of fatty acid composition of oils on flavor and stability of fried foods. J. Am. Oil Chem. Soc., v. 74, n. 4, p. 347356, 1997.

[30] WHITE, P.J. Métodos para medir los cambios en los aceites de fritura por inmersión en grasas. Alimentaria, n. 9, p. 81-87, 1991.

[31] ZHANG, W.B.; ADDIS, P.B. Prediction of levels of cholesterol oxides in heated tallow by dielectric measurement. J. Food Science, v. 55, p. 1673-1675, 1990.

\section{6 - AGRADECIMENTOS}

À Empresa Perdigão Agroindustrial S.A., pela doação dos produtos pré-fritos congelados utilizados neste estudo. 